\title{
Fatores que interferem no processo de ensino e aprendizagem
}

\author{
Factors that interfere in the teaching and learning process \\ Factores que interfieren en el proceso de enseñanza y aprendizaje
}

Recebido: 13/05/2021 | Revisado: 22/05/2021 | Aceito: 29/05/2021 | Publicado: 01/06/2021

\author{
Joniery Rubim de Souza \\ ORCID: https://orcid.org/0000-0002-0293-2886 \\ Instituto Federal de Educação, Ciência e Tecnologia do Maranhão, Brasil \\ E-mail:joniery@ifma.edu.br \\ Ariana de Oliveira Vital da Silva \\ ORCID: https://orcid.org/0000-0002-4854-087X \\ Universidade Estadual do Maranhão, Brasil \\ E-mail: arianavital@ professor.uema.br
}

\begin{abstract}
Resumo
A compreensão sobre os diferentes conceitos de aprendizagem, tal como a relação entre ensino e aprendizagem é de extrema importância para a educação, uma vez que a aprendizagem é discutida por diferentes teóricos da educação. Isso posto, o processo de ensino e aprendizagem se relaciona à interação entre professores e alunos no contexto educacional e durante tal processo podem emergir situações que podem afetar o ensino, bem como a aprendizagem. Deste modo, a presente pesquisa tem por objetivo identificar os elementos que podem interferir no processo ensino e aprendizagem, apresentando os fatores internos e externos que contribuem para tais interferências. A pesquisa se classifica como de natureza básica, com abordagem metodológica de caráter quanti-qualitativo e quanto aos seus objetivos se constitui em bibliográfica e exploratória. Foi realizado um levantamento na base de dados da CAPES e Google Acadêmico, visando subsidiar as seguintes questões: quais os fatores que podem interferir no processo de ensino e aprendizagem? Quais são as influências destes fatores no espaço escolar? Ao final da realização da pesquisa foi possível identificar que alguns dos fatores que causam danos à aprendizagem podem estar relacionados a questões emocionais, sociais e familiares.
\end{abstract}

Palavras-chave: Ensino e aprendizagem; Transtornos emocionais; Contexto escolar.

\begin{abstract}
Understanding the different concepts of learning, such as the relationship between teaching and learning, is of utmost importance for education since learning is discussed by different educational theorists. That said, the teaching and learning process is related to the interaction between teachers and students in the educational context, and during this process situations may emerge that can affect teaching as well as learning. Thus, this research aims to identify the elements that may interfere in the teaching and learning process, presenting the internal and external factors that contribute to such interference. The research is classified as basic in nature, with a quanti-qualitative methodological approach, and its objectives are bibliographic and exploratory. A survey was carried out in the CAPES and Google Academic databases, aiming to subsidize the following questions: which factors may interfere in the teaching and learning process? What are the influences of these factors in the school space? At the end of the research, it was possible to identify that some of the factors that cause damage to learning may be related to emotional, social, and family issues.
\end{abstract} Keywords: Teaching and learning; Emotional disorders; School settings.

\section{Resumen}

La comprensión de los diferentes conceptos de aprendizaje, como la relación entre la enseñanza y el aprendizaje, es extremadamente importante para la educación, ya que el aprendizaje es discutido por diferentes teóricos de la educación. Dicho esto, el proceso de enseñanza y aprendizaje está relacionado con la interacción entre profesores y alumnos en el contexto educativo y durante este proceso pueden surgir situaciones que pueden afectar tanto a la enseñanza como al aprendizaje. Así, esta investigación pretende identificar los elementos que pueden interferir en el proceso de enseñanza y aprendizaje, presentando los factores internos y externos que contribuyen a dicha interferencia. La investigación se clasifica como de carácter básico, con un enfoque metodológico de carácter cuanti-cualitativo y en cuanto a sus objetivos es bibliográfica y exploratoria. Se realizó una encuesta en las bases de datos CAPES y Google Académico, con el objetivo de subvencionar las siguientes preguntas: ¿qué factores pueden interferir en el proceso de enseñanza y aprendizaje? ¿Cuáles son las influencias de estos factores en el espacio escolar? Al final de la investigación se pudo identificar que algunos de los factores que causan daños en el aprendizaje pueden estar relacionados con cuestiones emocionales, sociales y familiares.

Palabras clave: Enseñanza y aprendizaje; Trastornos emocionales; Contexto escolar. 


\section{Introdução}

A compreensão sobre os diferentes conceitos de aprendizagem, tal como a relação entre ensino e aprendizagem é de extrema importância para a educação, uma vez que a aprendizagem é discutida por diferentes teóricos, entre eles teóricos da psicologia. Deste modo, Schmitt e Domingues (2016) apontam que o sujeito durante a sua trajetória de vida e na busca da construção de seu conhecimento apresenta diferentes técnicas para aprender.

Piovesan et al., (2018) destacam que grandes autores buscam compreender e descrever estilos de aprendizagem adotados pelo sujeito e destacam um modelo de aprendizagem baseado nos sentidos que podem ser compreendidos como estilo de aprendizagem visual, no qual o sujeito se utiliza de estímulos visuais para adquirir novos conhecimentos, estilo auditivo que leva em consideração o ato de ouvir e falar, seguido do estilo cinestésico cujo indivíduo aprende com base nos movimentos corporais.

Com base no exposto, Pereira (2010) destaca que o indivíduo por ser subjetivo adquire novos conhecimentos de acordo com suas potencialidades, aptidões, entre outros aspectos. Não obstante, a autora destaca a importância dos estímulos internos e externos que não só potencializam o ato de aprender, mas também oportunizam a motivação humana. Neste sentido, Camargo, Camargo \& Sousa (2019) destacam a importância da motivação, ressaltando que é por meio da motivação que os alunos, dentro do contexto educacional, podem adquirir e colocar em prática novas habilidades.

Para mais, a motivação humana é apresentada como um dos processos psicológicos básicos e provém de fatores internos e externos ao indivíduo. Além disso, tal processo pode ser considerado como um fator que impulsiona o indivíduo a desenvolver determinadas ações. No que se refere à educação, a motivação por parte dos alunos é fundamental, levando-se em consideração que eles quando motivados apresentam um maior engajamento nas atividades desenvolvidas tanto na sala de aula, quanto em todo o contexto educacional (Camargo, Camargo \& Sousa, 2019).

Cabe ressaltar que o aluno além de se diferenciar do outro, no âmbito da aquisição de conhecimentos, pode apresentar dificuldades durante a sua trajetória escolar, estas dificuldades podem surgir a partir de questões emocionais, psicomotoras ou até mesmo familiares. Tais dificuldades em grande parte podem exercer influências no processo de ensino e aprendizagem (Jacometo \& Tabile, 2017).

Por conseguinte, Bezerra (2014) aponta o fracasso escolar como uma consequência das dificuldades enfrentadas no decorrer do processo de ensino e aprendizagem. Ademais, a autora destaca que um dos fatores que leva o aluno ao fracasso escolar é a falta de motivação. Deste modo, compreende-se a importância da motivação durante tal processo, uma vez que ela oportuniza o aprendizado e contribui para que o indivíduo avance nas etapas. Assim sendo, o presente estudo propõe identificar os elementos que podem interferir no processo ensino e aprendizagem, apresentando os fatores internos e externos que contribuem para tais interferências.

\section{Referencial teórico}

A aprendizagem ocorre de forma dinâmica, sendo ela caracterizada por diferentes vertentes psicológicas, entre elas a teoria Behaviorista que aponta a aprendizagem como um resultado entre as experiências vividas pelo ser humano e os estímulos do meio. Pinto (2003) ao abordar a teoria Behaviorista aponta que a aprendizagem ocorre após a identificação de estímulos ofertados pelo meio que o indivíduo está inserido, ou seja, ao identificar tais estímulos o indivíduo emite respostas.

Neste sentido, Oliveira (2014) destaca que o indivíduo pode identificar estímulos do meio e adquirir novas aprendizagens desde a sua concepção, ou seja, o indivíduo é capaz de identificar estímulos a partir da sua vida uterina. A aprendizagem humana permanece em todas as fases do desenvolvimento e é por meio desta que o sujeito aprende o que é necessário para a sua sobrevivência, como aprender a se comunicar, se alimentar, respeitar os que estão a sua volta, entre outros fatores importantes.

Para mais, cabe citar a Epistemologia Genética de Jean Piaget que apresenta uma concepção de conhecimento pautada pela 
interação com o meio, ou seja, o sujeito passa a adquirir novos conhecimentos a partir da interação com o meio em que está inserido. Em consonância, Nunes e Silveira (2015) afirmam que para Piaget a construção do conhecimento é um processo contínuo e se forma a partir da relação ativa do sujeito com o meio físico e social.

Dentro desse contexto, Oliveira (2014) apresenta o inatismo e descreve que esta corrente teórica sustenta que o conhecimento do sujeito resulta da capacidade inata, ou seja, para o inatismo o homem vive em sociedade por apresentar instinto à sociabilidade. Para tal corrente teórica, ao nascer o sujeito traz em sua herança genética capacidades básicas.

Isso posto, Nunes e Silveira (2015) descrevem que para Vygotsky a aprendizagem se relaciona com a apropriação de conhecimentos, habilidades, valores e demais aspectos que incorporam a troca ativa do sujeito com o mundo em que está inserido. O teórico descreve dois tipos de aprendizagem de conceitos, sendo um deles, conceitos adquiridos por meio das experiências cotidianas da criança, como identificar o papel de cada membro da família ou até mesmo compreender a função de objetos domésticos. Do mesmo modo, apresenta o tipo de aprendizagem científica, que envolve os conceitos adquiridos por meio do ensino.

Não obstante, Nunes e Silveira (2015) descrevem que a aprendizagem pode ser observada através das primeiras experiências e manifestações na infância. Além disso, o processo de aprendizagem ocorre de inúmeras formas, uma vez que o sujeito não assimila os estímulos do meio da mesma forma. Diante disso, Schmitt e Domingues (2016), salientam que as diferentes concepções de aprendizagem têm ganhado espaço no âmbito educacional, em especial entre os docentes.

Por conseguinte, Nunes e Silveira (2015) introduzem a teoria das inteligências múltiplas de Howard Gardner, tal teoria sustenta que a competência cognitiva do indivíduo pode ser descrita como um conjunto de habilidades, talentos ou capacidades caracterizadas como inteligência. Logo, o teórico apresenta sete tipos de inteligências, sendo elas: linguística, lógico-matemática, corporal-cinestésica, espacial, musical, interpessoal e intrapessoal.

Neste seguimento, o sujeito adota diferentes estilos de aprendizagem e estes o auxiliam na assimilação de informações, no aprimoramento e aquisição de novas habilidades, entre outros aspectos. Apesar disso, é possível destacar que os estilos já citados fazem parte do processo de ensino, levando em consideração que o indivíduo, enquanto aluno, se utiliza de suas habilidades e competências para processar novas informações (Schmitt \& Domingues, 2016).

Portanto, cabe ressaltar que o processo de ensino e aprendizagem não é somente caracterizado por conquistas, Jacometo e Tabile (2017) descrevem que durante o processo de ensino podem emergir situações que são capazes de afetar o aluno e acarretar prejuízos à aprendizagem. Assim sendo, Silva e Hengemuhle (2019) apontam que dentro do contexto escolar podem surgir inúmeras situações que podem interferir no processo de ensino e aprendizagem, entre eles a indisciplina, falta de acompanhamento por parte da família, falta de material adequado e déficits de atenção.

De acordo com Torres et al. (2016) os fatores que podem provocar dificuldades na aprendizagem estão relacionados a fatores de ordem comportamental, emocional e neurológica. Desse modo, para que tais fatores não ocasionem danos ao aluno, é de extrema importância que os pais, professores e toda a comunidade escolar estejam atentos ao que é demandado pelo aluno, levando em consideração que quando tais fatores não são percebidos ou são negligenciados podem provocar danos irreparáveis.

Conquanto, é possível destacar que além dos aspectos citados, os transtornos emocionais e de aprendizagem tem se apresentado como fatores presentes no contexto educacional e que podem causar grandes prejuízos. Segundo Arduvino (2007), os transtornos emocionais podem estar relacionados a conflitos internos ou externos que causam sofrimento ao sujeito, entre outros aspectos, eles podem estar relacionados aos pensamentos acelerados, sentimentos e comportamentos desajustados que podem acarretar dificuldades na atenção, concentração e aprendizagem. Além disso, os transtornos psicológicos podem acarretar problemas na área motora ou cognitiva e sensorial.

Na sequência, Nunes e Silveira (2015) relatam que os transtornos de aprendizagem podem ser compreendidos como uma condição neurológica que afeta a aprendizagem e o processamento de informações, além disso, podem exercer influências no 
campo da linguagem, da leitura, da escrita e das habilidades matemáticas. Tais transtornos são caracterizados como dislexia, disgrafia, disortografia, dislalia e discalculia.

Ademais, além dos transtornos de aprendizagens podem surgir fatores que provocam dificuldades de aprendizagem. Portanto, é relevante pontuar a diferença entre dificuldades e transtornos de aprendizagem, uma vez que, os transtornos se caracterizam como duradouros e podem causar prejuízos ao aluno em toda sua trajetória escolar, diferentemente das dificuldades que são consideradas como inconstantes e podem surgir de acordo com experiências vivenciadas pelo aluno. Sobre este ponto, Oliveira (2014) destaca que os transtornos apresentam como características disfunções neurológicas, diferentemente das dificuldades de aprendizagem.

O mesmo autor destaca ainda que apresentar dificuldades de aprendizagem em determinados momentos da vida não significa que o aluno está com sinais de transtornos de aprendizagem, tendo em vista que o indivíduo pode apresentar tais dificuldades em distintos momentos. Todavia, elas não se prolongam em todas as fases do desenvolvimento e em outras situações, e o indivíduo pode assimilar novos conhecimentos.

A partir disso, as dificuldades de aprendizagem podem emergir com base em questões emocionais e estarem relacionadas a sintomas de ansiedade, mudanças de humor, ou fatores ligados ao contexto familiar, que envolvem os laços afetivos e o suporte dos pais ou responsáveis que é dado ao aluno, além de fatores culturais, psicomotores, cognitivos, entre outros aspectos (Oliveira, 2014).

Em vista disso, Sousa (2009) destaca que as dificuldades de aprendizagem que podem surgir por parte do aluno não decorrem somente da forma em que o professor aborda um conteúdo em sala de aula ou da metodologia utilizada, mas também de fatores existentes como os já mencionados que podem afetar o aluno. A autora pontua que é possível identificar com frequência os alunos que apresentam certa rejeição para aprender e diante de todas as questões apontadas, a busca pela identificação das potencialidades do aluno é algo que pode auxiliar o professor.

Jacometo e Tabile (2017) apontam que o professor exerce um papel importante ao identificar as dificuldades apresentadas pelo aluno, uma vez que, durante o seu desenvolvimento ele demonstra as dificuldades que podem emergir. Logo, Oliveira (2014) salienta que identificar as dificuldades de aprendizagem no contexto escolar é mais simples para o professor do que identificar possíveis sinais de transtorno de aprendizagem.

As autoras destacam que o processo de ensino só tem significado quando proporciona aprendizagem ao sujeito. Neste sentido, é de grande importância compreender quais são as potencialidades e limitações do aluno inserido no contexto escolar, para assim identificar como ele aprende o que é trabalhado em sala, tal como compreender de que forma o professor aborda o conteúdo em sala.

\section{Metodologia}

A presente pesquisa é de natureza básica, de abordagem metodológica quanti-qualitativa, por meio bibliográfico e exploratório. Inicialmente foi realizada uma revisão das fontes bibliográficas com o objetivo de coletar informações sobre a temática de estudo. Assim, Lima e Mioto (2007) descrevem que a pesquisa bibliográfica pode ser caracterizada como um processo que se utiliza de ferramentas para buscar dados e todas as informações necessárias que possam responder aos objetivos de determinados estudos e pesquisas.

Para tanto, a pesquisa em questão foi subsidiada de publicações extraídas das bases de dados da CAPES e do Google Acadêmico. A partir dos materiais levantados, foi possível realizar uma busca por dados que contemplassem as questões que norteiam essa pesquisa e fazer uma análise dos elementos identificados que foram divididos em categorias para melhor discussão dos resultados. 
Nessa perspectiva, visando identificar e selecionar os materiais mais relevantes para a pesquisa e posteriormente realizar a análise dos dados coletados, foram utilizados os termos: processo de ensino e aprendizagem, dificuldades de aprendizagem, e problemas que podem interferir no processo ensino e aprendizagem.

\section{Resultados e Discussão}

Por meio das pesquisas realizadas nas bases de dados da CAPES e do Google Acadêmico, foi possível identificar e selecionar 19 produções publicadas entre os anos de 2003 a 2019. Com a finalidade de quantificar as produções, foi estruturada uma tabela (Tabela 1) para classificá-las por ano.

De acordo com Souza (2017), a base de dados da CAPES foi criada em 1990 com o objetivo de beneficiar o ensino de pósgraduação, proporcionando acesso a inúmeros materiais, entre eles: livros e periódicos em geral, constituindo-se de grande relevância no meio científico com grandes contribuições para todas as áreas. A Tabela 1 traz as produções selecionadas e seus respectivos anos de publicação para execução do estudo.

Tabela 1: Quantitativo de Produções por ano.

\begin{tabular}{c|c|c|c|c|c|c|c|c|c}
\hline Ano & $\mathbf{2 0 0 3}$ & $\mathbf{2 0 0 6}$ & $\mathbf{2 0 0 7}$ & $\mathbf{2 0 1 0}$ & $\mathbf{2 0 1 4}$ & $\mathbf{2 0 1 6}$ & $\mathbf{2 0 1 7}$ & $\mathbf{2 0 1 8}$ & $\mathbf{2 0 1 9}$ \\
\hline ARTIGOS & & 1 & 1 & 1 & 2 & 4 & 2 & 1 & 3 \\
\hline LIVROS & 1 & & & & & & & & \\
\hline MONOGRAFIAS & & 1 & 1 & & 1 & & & & \\
\hline
\end{tabular}

Fonte: Autoria própria (2020).

Com base na Tabela 1, percebe-se que foi selecionado um (1) livro no ano de 2003 (clássico), dois (2) trabalhos no ano de 2006, sendo um (1) artigo e uma (1) monografia; no ano seguinte, em 2007 foram dois (2), sendo um (1) artigo e (1) monografia; nos anos posteriores, 2010 foi encontrado um (1) artigo, em 2014 foram três (3) trabalhos, sendo dois (2) artigos e uma (1) monografia, 2016 quatro (4) artigos, 2017 dois (2) artigos, 2018 um (1) artigo e em 2019 três (3) artigos.

De acordo com o levantamento realizado nas bases de dados CAPES e do Google Acadêmico foram identificadas as às seguintes produções como mostra o Quadro 1:

Quadro 1: Teses por ano, autoria, título e instituição de ensino.

\begin{tabular}{|c|c|c|c|}
\hline ANO & AUTORIA & TITULO & IE \\
\hline 2003 & Pinto, G. & $\begin{array}{l}\text { Psicologia da aprendizagem: Concepções, Teorias e } \\
\text { Processos }\end{array}$ & IEFP \\
\hline 2006 & Lemes, R. P. \& Alexandre, S. & $\begin{array}{c}\text { Os fatores que interferem no processo de ensino e } \\
\text { aprendizagem }\end{array}$ & UniCEUB \\
\hline 2007 & $\begin{array}{l}\text { Lima, T. C. S. \& Mioto, R. C. } \\
\text { T. }\end{array}$ & $\begin{array}{l}\text { Procedimentos metodológicos na construção do } \\
\text { conhecimento científico: a pesquisa bibliográfica }\end{array}$ & UFSC \\
\hline 2009 & Sousa, S. M. S. & $\begin{array}{c}\text { Aprender - não aprender: os múltiplos fatores que } \\
\text { interferem nesse processo. }\end{array}$ & UEG - UnU \\
\hline 2010 & Pereira, D. S. C. & $\mathrm{O}$ ato de aprender e o sujeito que aprende & UCS - FE \\
\hline 2014 & Oliveira, F. G. & Psicologia da Educação e da Aprendizagem & UNIASSEL VI \\
\hline 2014 & Bezerra, L. C. M. & $\begin{array}{c}\text { Os fatores que interferem no processo de ensino e } \\
\text { aprendizagem }\end{array}$ & UEPB \\
\hline 2015 & $\begin{array}{l}\text { Nunes, A. I. B. L. \& Silveira, } \\
\text { R. N. }\end{array}$ & Psicologia da Aprendizagem & EdUECE, \\
\hline
\end{tabular}


Research, Society and Development, v. 10, n. 6, e29210616071, 2021

(CC BY 4.0) | ISSN 2525-3409 | DOI: http://dx.doi.org/10.33448/rsd-v10i6.16071

\begin{tabular}{|c|c|c|c|}
\hline 2017 & Arduvino, A. M. & $\begin{array}{c}\text { Dificuldades de aprendizagem, transtornos de } \\
\text { aprendizagem e deficiência mental. Do que estamos } \\
\text { falando? }\end{array}$ & UFSM \\
\hline 2017 & Souza, J. A. & Busca de informações em bases de dados digitais & UAB UFMG \\
\hline 2017 & $\begin{array}{l}\text { Jacometo, M. C. D. \& Tabile, } \\
\text { A. F. }\end{array}$ & $\begin{array}{l}\text { Fatores influenciadores no processo de aprendizagem: um } \\
\text { estudo de caso }\end{array}$ & $\begin{array}{l}\text { Rev. } \\
\text { Psicopedago } \\
\text { gia }\end{array}$ \\
\hline 2016 & $\begin{array}{c}\text { Torres, N. L., Soares, T. S. \& } \\
\text { Conceição, F. H. G. }\end{array}$ & Dificuldade de aprendizagem: além do muro escolar & FAMA \\
\hline 2016 & Alves et Al. & $\begin{array}{l}\text { Fatores que influenciam a aprendizagem de conceitos } \\
\text { matemáticos em cursos de engenharia: Um estudo } \\
\text { exploratório com estudantes da Universidade do Minho }\end{array}$ & $\begin{array}{l}\text { Universidad e do } \\
\text { Minho } \\
\text { - Portugal }\end{array}$ \\
\hline 2016 & $\begin{array}{c}\text { Schmitt, C. S. \& Domingues, } \\
\text { M. J. C. S }\end{array}$ & Estilos de aprendizagem: um estudo comparativo. & $\begin{array}{l}\text { Revista da } \\
\text { Avaliação da } \\
\text { Educação } \\
\text { Superior } \\
\text { (Campinas) }\end{array}$ \\
\hline 2019 & $\begin{array}{l}\text { Camargo, C. A., Cardoso, M. } \\
\text { Camargo, M. A. F. \& Souza, } \\
\text { V. O. }\end{array}$ & $\begin{array}{l}\text { A importância da motivação no processo } \\
\text { Ensino aprendizagem }\end{array}$ & UEMG \\
\hline 2019 & $\begin{array}{l}\text { Silva, V. H. S. \& } \\
\text { Hengemuhle, A. }\end{array}$ & $\begin{array}{c}\text { Análise dos fatores que interferem na aprendizagem de } \\
\text { alunos do } 1^{\circ} \text { ao } 3^{\circ} \text { do Ensino Fundamental no } \\
\text { município de Ocara }\end{array}$ & Rev. Mult. Psic \\
\hline 2019 & $\begin{array}{l}\text { Coutinho, N. R., Damasceno, } \\
\text { S. C.A. \& Damasceno, } \\
\text { S.C. }\end{array}$ & $\begin{array}{l}\text { Fatores didáticos que interferem direto ou indireto no } \\
\text { ensino aprendizagem da matemática do ensino } \\
\text { fundamental. }\end{array}$ & UTIC \\
\hline 2016 & $\begin{array}{l}\text { Pavioni, C. S. S. N., Avelino, } \\
\text { B. C. \& Francisco, J. R. S. }\end{array}$ & $\begin{array}{c}\text { Fatores que influenciam o processo de } \\
\text { ensinoaprendizagem sob a perspectiva de estudantes do } \\
\text { curso de ciências contábeis: Análise em uma instituição } \\
\text { de ensino superios de Minas Gerais }\end{array}$ & REPEC \\
\hline
\end{tabular}

Fonte: Autoria própria (2020).

Com base na bibliografia já citada, é possível identificar que o assunto: fatores que interferem no processo de ensino e aprendizagem é um tema muito estudado atualmente. Deste modo, Lemes e Alexandre (2006) apontam que ao buscar compreender os fatores que interferem na aprendizagem é necessário levar em consideração aspectos relacionados ao contexto social em que a criança está inserida, contexto familiar, escola, sociedade, entre outros aspectos que podem interferir na aprendizagem.

Diante disso, Oliveira (2014) destaca que a sociedade ao longo dos anos tem passado por diversas transformações, mudanças que envolvem a ciência e a tecnologia, além disso, diversas mudanças têm afetado a família, o mercado de trabalho, fatores culturais, dentre outros aspectos. Outrossim, é necessário saber lidar com todas essas questões e buscar novos conhecimentos para saber se colocar diante das transformações que estão ocorrendo na sociedade.

Por consequência, a literatura evidencia que um dos fatores que podem interferir no processo de ensino e aprendizagem é a desmotivação. Neste seguimento, Jacometo e Tabile (2017) destacam que um dos fatores que podem estar relacionados à desmotivação é a metodologia utilizada pelo professor. Neste contexto, Alves et al. (2016) afirma que a forma como os alunos assimilam o conteúdo e adquirem novas aprendizagens ocorre de diferentes maneiras, assim sendo é necessário que o professor adote metodologias de ensino dinâmicas e variadas.

Assim sendo, Lemes e Alexandre (2006) enfatizam que nos anos anteriores não era levado em consideração o fator desmotivacional, apresentado pelo indivíduo durante o processo de ensino e aprendizagem. Quando as crianças demandavam problemas relacionados à dificuldade de aprendizagem, eram direcionadas para ambientes educacionais onde eram aplicadas metodologias de ensino diferenciadas. Os autores pontuam que nem todos os fatores que surgem durante o processo de ensino e 
aprendizagem devem ser considerados dificuldades de aprendizagem, uma vez que existem crianças que não apresentam deficiência, entre outros problemas de aprendizagem, no entanto, podem levar um tempo maior para aprender.

Neste sentido, Silva e Hengemühle (2019) indicam que os alunos podem demonstrar diferentes demandas relacionadas as dificuldades dentro do contexto escolar, entre as dificuldades citadas pelos autores estão à indisciplina, falta de atenção (considerada um dos elementos mais presentes no contexto educacional), dificuldade de compreensão, a ausência de ambiente escolar adequado, a falta de estímulo por parte dos pais e a falta de organização em relação aos materiais escolares. Pavione, et al. (2016) descrevem que a falta de interesse, as conversas em sala de aula e a falta de compromisso em relação a entrega de atividades são também fatores que podem interferir negativamente no processo de ensino e aprendizagem.

Isso posto, Torres et al. (2016) descreve que além dos fatores já citados, um dos mais comuns que podem causar danos à aprendizagem é a hiperatividade, ou seja, crianças que apresentam esse transtorno podem apresentar dificuldade na aprendizagem. Assim sendo, é possível identificar em crianças hiperativas, inquietação na sala de aula, conversas constantes e dificuldade de aguardar sua vez para participar de determinadas atividades.

Deste modo, Oliveira (2014) aponta que fatores emocionais, familiares, psicomotores, culturais, e entre outros, podem influenciar na aprendizagem. Além disso, a autora pontua que mudanças sociais, a forma como o indivíduo se relaciona, ações e até mesmo expressões podem acarretar dificuldades. Ademais, ao buscar compreender as dificuldades que podem surgir no contexto escolar, é preciso levar em consideração não somente o que é apresentado pelo aluno como um todo, mas também a sua estrutura familiar.

Coutinho et al. (2019) ressalta que as necessidades demandadas por parte dos alunos são presentes no contexto escolar e para lidar com tais dificuldades é necessário que o educador coloque em prática sua criatividade. $\mathrm{O}$ autor descreve que os fatores que podem prejudicar no processo de aprendizagem estão relacionados aos retardos mentais, transtornos de aprendizagem, distúrbios físicos e emocionais.

Além disso, o autor também descreve que é possível identificar com frequência na educação infantil, problemas relacionados à linguagem, à falta de atenção e às dificuldades de concentração. À vista disso, Torres et al. (2016) aponta que os fatores que envolvem a linguagem e que podem ocasionar dificuldades na aprendizagem se relacionam com as palavras expressadas pelos alunos e podem prejudicar na leitura e na escrita.

Desta maneira Torres et al. (2016) descreve que quando a criança recebe o suporte dos pais e dos professores, as dificuldades que surgem na trajetória escolar podem ser ressignificadas, isso acontece quando tais dificuldades são consideradas temporárias e se relacionam à adaptação do aluno. Porém, existem dificuldades que podem afetar o aluno em toda a trajetória escolar e no seu desenvolvimento, portanto, é necessário que o professores e os pais estejam atentos para auxiliar o aluno.

Em suma, é necessário que tanto os pais e responsáveis quanto os educadores estejam atentos aos alunos e ao seu desenvolvimento, tendo em vista que é possível identificar as dificuldades apresentadas pelos indivíduos e uma vez que as dificuldades de aprendizagem são identificadas, é possível desenvolver ações e atividades que dão suporte a todos os alunos. Isto posto, Torres, Soares \& Conceição (2016) afirmam que a partir do momento que é identificado os fatores já citados, os pais exercem um papel de grande importância no crescimento dos filhos na vida escolar.

\section{Considerações Finais}

O presente estudo objetivou identificar e apontar os fatores que interferem no processo de ensino e aprendizagem. Para tanto, foi realizada uma pesquisa bibliográfica e, por meio desta, foi possível identificar que os fatores que interferem no processo de ensino e aprendizagem são inúmeros, sendo que tais fatores podem ocorrer de forma provisória e outros podem permanecer em todas as fases do desenvolvimento do sujeito. 
Com base no exposto, o material analisado apontou que os fatores que interferem no processo de ensino e aprendizagem estão relacionados à motivação, à falta de atenção, à hiperatividade e à problemas de linguagem que podem interferir tanto na leitura quanto na escrita, dentre outros fatores discutidos neste artigo. Além disso, durante a realização da pesquisa foi possível identificar que os fatores que causam danos à aprendizagem podem estar relacionados à questões emocionais, sociais e familiares.

Posto isso, a partir do estudo realizado identificou-se a importância do papel dos professores neste processo frente as dificuldades. Tabile e Jacometo (2017) orientam que o ensino só é levado em consideração quando este proporciona a aprendizagem humana e no meio educacional se faz necessário compreender como ocorre o ensino por parte do professor e como o aluno assimila as novas informações e conhecimentos apresentados durante as aulas.

\section{Referências}

Alves et al. (2016). Fatores que influenciam a aprendizagem de conceitos matemáticos em cursos de engenharia: Um estudo exploratório com estudantes da Universidade do Minho. Rev. Portuguesa de Educação. p, 259 - 293.

Arduvino, A. M. (2007). Dificuldades de aprendizagem, transtornos de aprendizagem e deficiência mental. Do que estamos falando? S'ant Ana do Livramento, RS

Bezerra, L. C. M. (2014). Dificuldades de aprendizagem e os fatores que influenciam o fracasso escolar. Monografia (Especialização em práticas pedagógicas interdisciplinares). Universidade Estadual da Paraíba. Itaporanga, PB. P. 53.

Camargo, C. A. C. M., Camargo, M. A. F. \& Souza, V. O. (2019). A importância da motivação no processo ensino-aprendizagem. Rev. Thema, v. 16, n.3, p. 598606.

Coutinho, N. R., Damasceno, S. C. A. \& Damasceno, S. C. (2019). Fatores didáticos que interferem direto ou indireto no ensino aprendizagem da matemática do ensino fundamental. Rev. Cesmac, v.8, n.10.

Jacometo, M. C. D. \& Abile, A. F. (2017). Fatores influenciadores no processo de aprendizagem: um estudo de caso. Rev. Psicopedagogia, p. 75 - 86.

Lemes, R. P. \& Alexandre, S. (2006). Os fatores que interferem no processo de ensino e aprendizagem. Monografia (Graduação em pedagogia). Faculdade de Ciências de Educação - FACE. Centro Universitário de Brasília. Brasília. P. 78.

Lima, T. C. S. \& Mioto, R. C. T. (2007). Procedimentos metodológicos na construção do conhecimento científico: a pesquisa bibliográfica. Rev. Katálysis, vol.10, Florianópolis.

Nunes, A. I. B. L. \& Silveira, R. N. (2015). Psicologia da Aprendizagem. 3. ed. rev. - Fortaleza: EdUECE.

Oliveira, F. G. (2014). Psicologia da Educação e da Aprendizagem. Uniasselvi. Indaial.

Pavioni, C. S. S. N., Avelino, B. C. \& Francisco, J. R. S. (2016). Fatores que influenciam o processo de ensino-aprendizagem sob a perspectiva de estudantes do curso de ciências contábeis: Análise em uma instituição de ensino superior de Minas Gerais. Rev. REPeC, Brasília, v. 10, n. 2, art. 5, p. 196-219.

Pereira, D. S. C. (2010). O ato de aprender e o sujeito que aprende. Rev. Construção Psicopedagógica, São Paulo, SP, v. 18, n.16, p. 112-128.

Pinto, G. (2003). Psicologia da Aprendizagem, Concepções, Teorias e Processos. 4. Ed. IEFP.

Piovesan et al. (2018). Psicologia do desenvolvimento e da aprendizagem. 1. ed. Santa Maria, RS: UFSM, NTE, UAB.

Schmitt, C. S., \& Domingues, M. J. C. S. (2016). Estilos de aprendizagem: um estudo comparativo. Rev. Avaliação. Campinas; Sorocaba, SP, v. 21, n. 2, p. 361385 .

Silva, V. H. S, Hengemuhle, A. (2019). Análise dos fatores que interferem na aprendizagem de alunos do $1^{\circ}$ ao $3^{\circ}$ do Ensino Fundamental no município de Ocara. Rev. Mult. Psic. v.13, n. 45, p. 502-532.

Sousa, S. M. S. (2009). Aprender - não aprender: os múltiplos fatores que interferem nesse processo. UEG - UnU. São Luís de Montes Belos, MG.

Souza, J. A. (2017). Busca de informações em bases de dados digitais. UAB-UFMG, Cuiabá, MT.

Torres, N. L., Soares, T. S. \& Conceição, F. H. G. (2016). Dificuldade de aprendizagem: Além do Muro Escolar. FAMA. <http://faculdadea madeus.com.br/graduação/Web/content/content-anais/encontromultidisciplinar/attachments/>. 